\title{
Neuromuscular Re-Education Using Surface Electromyography Biofeedback
}

\section{Magdalini Stamou*}

Physiotherapy Department, Laboratory of Neuromuscular and Cardiovascular Study of Motion, University of West Attica, Athens, Greece

*Corresponding Author: Magdalini Stamou, Physiotherapy Department, Laboratory of Neuromuscular and Cardiovascular Study of Motion, University of West Attica, Athens, Greece.
Received: July 26, 2021

Published: August 12, 2021

(C) All rights are reserved by Magdalini

Stamou.

\begin{abstract}
This paper investigates the role of Surface Electromyography Biofeedback in neuromuscular re-education. It references the theoretical basis of biofeedback action, the physiological mechanism of the peripheral motor neuron, recruitment and modification of central stimulation and brain plasticity. This paper includes an analysis of the technical characteristics of surface electromyography as well as of the advantages and disadvantages of these applications.
\end{abstract}

Keywords: Surface Electromyography Biofeedback; Neuromuscular Re-Education; Electrophysical Agents; Assessment; Electrotherapy; Brain Plasticity

\section{Abbreviations}

SEMG BFB: Surface Electromyography Biofeedback; ATI: AptitudeTreatment-Interaction; SMUT: Single Motor Unit; PET: Positron Emission Tomography; MRI: Magnetic Resonance Imaging; f-MRI: Functional Magnetic Resonance Imaging

\section{Introduction}

The SEMG BFB was made known to scientific community from the 1960. Historically, SEMG and Biofeedback are related to the discovery of electricity and tools that can detect electric currents in living beings. In 1600 Francesco Redi observed that a specialized muscle of a fish was responsible for the light emitted by it. The revolution started when Galvani, in 1790, associated the muscle contraction with electricity. In 1792, Volta agrees with Galvani's findings and concludes that the electric currents observed in the muscle was not due to the muscle itself but due to the metals that came in contact with it. Many decades of research were needed for Galvani to prove its findings as Volta was more famous and influential. Later, Volta built a tool that produced electric currents which stimulated the muscle tissue. Stimulation of muscle tissue by electric currents has intrigued many researchers during the $19^{\text {th }}$
Century, where it was used for research purposes. In 1800 the galvanometer was invented, a tool that allowed the measurement of electrical waves and muscle activity. In 1890, Duchenne conducts the first systematic review regarding the relation between the electrical potential difference and muscle activity using electrical muscle stimulation. In 1912, the history of electromyography begins with Piper H. conducting a research on the electromyographical signals by using the galvanometer. In 1920, Gasser and Newcomer using the newly discovered oscilloscope show the signals that the muscles receive. As such they win the Nobel Prize of 1944 [1-3].

There have been studies that underpin the scientific basis of electromyography, which is now used for neurophysiological studies on motion control and movement education.

Biological feedback or biofeedback is the recording of physiological events occurring in the human body which are not perceived, and how the human body becomes aware of these events. In particular, biofeedback affects the neuromuscular system and the autonomic nervous system. It records physiological and nonphysiological functions by means of electronic and electromechanical equipment and informs the patient and the therapist visually auditorily [4]. 
The theoretical basis of SEMG BFB

Biofeedback is a form of operant conditioning and is used for the reinforcement (or destruction) of responses that are not known to the patient. Operant conditioning, one of the two main models of associative learning, concerns the modification of active, responsive behaviours with the help of a stimulus, following the law of effect. In other words, behaviour is reinforced when it causes favourable changes in the environment. An example could be when there are slight muscular contractions in a stroke patient or peripheral nerve neurotisations. The contractions are recorded with an electronic device that transmits direct visual or auditory stimuli and the patient's responses to these stimuli. If the patient wants to increase the frequency or strength of the responses, the feedback signal acts as positive reinforcement [3,5-9].

The most important theoretical models mentioned in the literature are:

1. Physiological changes

2. Feed-forward processes

3. Cognitive changes

4. Placebo non-specific effects

5. Bandura's self-efficacy

6. Patient education model

7. The Rosenthal interpersonal expectancy

8. Aptitude-treatment-interaction (ATI).

Physiological changes

This model is based on the collection of information by external sensors and the awareness of the condition of the affected area through visual or auditory stimuli. This awareness initiates a closed-loop feedback process that drives the human body to respond to the external stimuli [5,7-9].

\section{Feed-forward processes}

This model relates to the process of setting a predetermined sequence of actions in anticipation of a particular result. It is based on external information that is updated and redefined by further information obtained from each site separately and simultaneously $[10,11]$.

\section{Cognitive changes}

Cognitive changes relate to internal restructuring through symbolism or imagery of the external environment and of the experiences that a person has undergone. Rather than being a technique of psychological intervention, cognitive behavioural therapy is a set of techniques.

Cognitive changes are based on three fundamental principles:

- $\quad$ Cognitive activity affects behaviour.

- $\quad$ Cognitive activity is monitored and modified.

- $\quad$ Desired behaviour may be due to cognitive changes.

The results of research conducted by M. O' Callaghan and B. Couvadelli (1998), using cognitive modification techniques, on patients with neurological disorders due to stroke and traumatic brain injury were very satisfactory for achieving kinetic and opticokinetic targets. The results of a research conducted by Rosen., et al. in 1989 on the negative image showed that patients that had of their bodies were statistically significantly different after cognitive therapy $[12,13]$.

\section{Placebo non specific effects}

In the $18^{\text {th }}$ century, The New Medical Dictionary defined placebo as 'a commonplace method or medicine' [14]. Shapiro was the first to define placebo as any treatment administered for non-specific psychological or psychophysiological purposes. It is scientifically accepted that approximately $1 / 3$ of a therapy is generally due to the placebo effect, and the time required by treatment to take effect is measured after 6 weeks [15]. Price., et al. 2007, states that the administration of a placebo treatment in a therapeutic context has different effects, depending on the context [16]. The action mechanisms of a virtual therapeutic approach could be divided into psychological and neurobiological mechanisms [17-19].

The clinical response to treatment, regardless of the administered drug or placebo, involves a common pattern of changes in specific cortical areas [19].

Citation: Magdalini Stamou. "Neuromuscular Re-Education Using Surface Electromyography Biofeedback". Acta Scientific Orthopaedics 4.9 (2021): 40 -48. 


\section{Bandura's self-efficacy}

Bandura's self-efficacy is people's conviction about their ability to influence events in their lives. Self-efficacy is determined by four central pillars: cognitive processes, motivation, affective processes and selection [20].

\section{Patient education model}

In recent years, there has been an emphasis on patient education and patient involvement in health decisions. It has been found that informing and educating patients has beneficial effects on their compliance, satisfaction and quality of life as well as on clinical results [21-23].

\section{The Rosenthal interpersonal expectancy}

The term interpersonal expectancy relates to a person who acts towards another and has specific expectations, regardless of whether they are realistic or not. It is how we evaluate an event that determines our behaviour, rather than what actually happens. The change in the behavioural model also determines the outcome; hence the name 'self-fulfilling prophecy' [24,25]. The first and most famous study on this theory was carried out by Rosenthal R. and Jacobson L. in 1968. The theory was later confirmed in a meta-analysis performed by Rosenthal and Rubin in 1978 with 345 studies. The qualitative analysis of the studies found a relatively low correlation between the experiments and the expectation but a statistically significant correlation between the effect of expectancy and the results [25].

\section{Aptitude * Treatment Interaction}

It is a cognitive learning theory. It refers to a structured model of education in which the trainees' skills and the development of training are based on the interaction between the instructor and the trainees. The model describes how to create a learning environment that suits the individual learner's competence. The skills and training regimen are two variables that interact with the environment and the instructor [26].

The anatomical and physiological basis of SEMG BFB

In during the 1960s, the biofeedback technique was born. Basmajian's work on single motor unit training provided some of the impetus for research on biofeedback. Although this type of training entailed the use of fine-wire electrodes rather than surface electrodes, it clearly demonstrated that EMG feedback could be used to train the neuromuscular system, based on its most basic element: the Single Motor Unit (SMUT) [27].

At the same time, Marinacci showed the correlation of biofeedback by means of electrodes that detected muscle function. The brain became aware of the muscle function and responded using reaction control. He also stated that any likely cerebral concentration functions could make up for muscle weaknesses in a short time [28].

Later in 1983, Wolf S. states that patient information obtained from SEMG BFD function:

a) Centrally, explaining how the received peripheral repetitive (visual or auditory or verbal) stimuli activated the brain centrally, using sub-functioning pathways or opening new ones. It was reported that there was feedback from peripheral stimuli due to brain plasticity and its ability to make changes $[29,30]$.

b) To override the injury and transmit information regarding muscle change to a higher level than that of the injury through somatosensory pathways [29,30].

c) As a bypass: Bypassing the defective route, opening a new somatosensory pathway and producing a new motor response in the brain stem $[29,30]$.

Brain plasticity

The human brain is the most complex and unexplored organ. The development of imaging techniques, such as positron emission tomography (PET), magnetic resonance imaging (MRI) and functional magnetic resonance imaging ( $\mathrm{f}$ - MRI), was able to detect the changes in activity of neural structures with specific cognitive and motor processes in the living human brain. The characteristic plasticity, that neural networks must alter their functions, chemical profiles, and structures is an essential process for the restoration of damage to the CNS [31].

The approach involves an internal representation that any perceptual or kinetic energy is associated with a characteristic type of neuronal activity in a specific set of interconnected cells, which is considered the cornerstone of brain science.

Citation: Magdalini Stamou. "Neuromuscular Re-Education Using Surface Electromyography Biofeedback". Acta Scientific Orthopaedics 4.9 (2021): $40-48$. 
Researchers have mapped functional regions that correspond to motor and sensory stimuli [32]. Not all parts of the body are equally represented. For example, the somatosensory representation of the face or the index finger is much larger than that of the bottom or back of the head and is related to the difference in innervation density from region to region. Also, those body parts used for tasks that require smooth and precise movements, such as the hand or face, occupy a proportionally larger area.

It was formerly believed that the cortical map was formed at birth and remained undifferentiated in later life. Over the past decades, research has confirmed that the cortical map is modified according to the stimuli received. So, a particular area can grow, as it happens to stringed-instrument musicians, or shrink in cases of inactivity [33-36].

In addition, in the initial training phase of a particular motion, large and diffuse areas of the brain engage in a synaptic activity. When this motion is mastered, the MRI results are differentiated. The cortical map shows smaller demarcated and specific areas [37].

Similarly, in cases of upper-limb amputations, the region of the amputated hand is now occupied by the face region, adjusting the response according to the stimulus [38]. Stimuli coming from the periphery show a different location. For example, a stimulus to the patient's chin gives a sense of numbness of the fifth mutilated finger.

The research conducted by Jiang., et al. (2010) on rats recorded the redefinition and reconstruction of the cortical map. Specifically, after brachial plexus injury, the primary kinetic map is identified before neurotisation. There are functional changes in the motor map before innervation. The inactive cerebral areas lose their representation and are occupied by adjacent areas in the primary motor cortex. The ability of the brain to change depending on the stimuli justifies and promotes the need for as many external stimuli as possible. Furthermore, the rehabilitation programme seems to affect brain reconstruction significantly $[39,40]$. Specifically, the implementation of specific activities, rather than a generalised exercise programme, and the shortest time of the rehabilitation programmes start have a particularly favourable effect on rehabilitation [40].
The re-education of the neuromuscular system is essentially presented as a hypothetical circular trip that starts from the CNS, giving a command to initiate the recording of the execution and return to patient adjustment. This whole part is at risk from potential errors that may occur at each neuromuscular junction.

\section{Peripheral activity}

Sherrington was the first to introduce the term 'motor unit' in 1925, it describes the basic unit of motor function that underlies all kinetic behaviour. The motor neuron (body and axon) and the muscle fibres innervated by the final nerve make up a motor unit. The number of nerve fibres innervated by a motor neuron is called innervation ratio. It varies from muscle to muscle and is proportional to the size of the motor neuron.

The nervous system can alter the strength of muscle contraction in two ways:

\section{Motor unit recruitment.}

\section{Change in the rate of firing.}

\section{Motor unit recruitment}

According to the principle of size, the larger the cell body, the greater the conduction velocity is. As a motor unit acts, the first weak impulses activate the lowest threshold and, as the power or the demand for power increases, the motor neurons with larger cell bodies operate progressively.

Motor units are activated in a specific order, from the weakest to the strongest ones. This gradual activation has two important functional consequences.

First, senior centers need only to determine the size of the synaptic potential to be transferred to the group of motor neurons as a whole and therefore do not require separate commands to trigger specific motor units.

Second, the slow motor units are the most widely used and metabolically the most economical for the human body. Whether all muscle fibres participate or not depends on the requested movement. The more increasing the need for work is, the more muscle fibres and motor neurons are active. 
The mechanism of changing the firing rate

The change in the rate of motor neuron depolarization alters muscle strength. The potential action in a nerve or a muscle lasts only 1 to $3 \mathrm{~ms}$ while a short muscle contraction time lasts from 10 to $100 \mathrm{~ms}$. Thus, increasing the depolarization frequency allows the dynamic energy to accumulate and yield a stronger contraction [41].

Muscle re-education with Surface EMG Biofeedback depends on this theoretical basis for recruitment and change of firing rate, triggering more muscle fibres and increasing the firing rate of motor neurons.

The technical characteristics of surface electromyography biofeedback

Every time that action potential is at a muscle fiber, a small part of the electric current is transmitted from muscle to skin. If many muscle fibers constrict simultaneously, electrical potentials added to the skin giving high graves. The EMG signals are the composition of the potential movement of the muscle fibers which are organized in motor units. This signal is detected by sensors placed on the skin or needles in muscle tissue [42].

Using surface electrodes, the direction of the electric charge is considered 'blurry' and cyclic since signals are received from more than one point and the average duration is about $5 \mathrm{~ms}$. The potential of motor unit appears in the cathode electrode of the oscilloscope as sharp spikes often displayed as a three-phase or twophase. The greater the potential energy, the larger the phenomenon of contraction. A change in muscle tension causes changes in the electromyographic recording (curvilinear number, width, rise time) $[43,44]$.

\section{The advantages and disadvantages of SEMG BFB}

The use of surface Electromyography offers a non-invasive (there is no skin penetration), safe and easy recording of muscle energy status. Surface electrodes provide the opportunity to study the motor unit action potentials changes at contraction and relaxation and obtain important information for muscles which could not be obtained via naked eye. By adding SEMG recording information to the practitioner's fund of knowledge about the muscle function and dysfunction and suggest methods to improve treatment approaches.
Difficulties that may present include:

- Topography: The neuromuscular structure is rich and complex, as it is as the entire human system and its recording presents practical difficulties. The topographical position of the muscle studied plays an important role, whether the specific muscle is superficial or deep, or is between the electrode and the muscle tissue adipose tissue exist or other muscles influencing the signals received by the sensorelectrode.

- The crosstalk: it is possible to record signals not only from the specific muscle examined but also from muscle that are anatomically very close. This phenomenon is called crosstalk. The phenomenon is influenced by anatomical conditions such as the thickness of the subcutaneous tissue and the detection system that is used.

One possible approach to reduce the crosstalk phenomenon is to place electrodes at different points on the skin and evaluate the findings. Again, the literature indicates that there may be unpredictable errors without assurance of perfect selectivity.

Another possible approach in eliminating crosstalk is the application of electrical stimulation. The signals received with simple contraction differ quantitatively from those obtained with electrically stimulated contraction. The application of electrical stimulation was first proposed by De Luca and Merletti [43,44].

Conclusively: a) crosstalk is mainly due to signals generated at the extinction of the potentials at the tendon regions, b) the crosscorrelation between signals remote from the active muscle and signals detected over the active muscle do not reflect the amount of crosstalk. c) crosstalk reduction by spatial or temporal filtering of EMG signals should be used with caution considering the nonpropagating nature of crosstalk. d) crosstalk signals contain high frequency components.

Other studies indicated that the surface EMG detection with appropriate electrodes size and appropriate distance between them reduces the crosstalk between adjacent muscles. This phenomenon must be taken into account where muscles are deep and where adipose tissue is interposed between the muscles and the electrode. Research studies which evaluated movement, e.g. walking, weight- 
lifting, the crosstalk phenomenon does not affect the recording and it is perceived as further external noise $[45,46]$.

Recent surveys indicate that the effect of crosstalk is negligible in EMG with the use of surface electrodes [47].

\section{Selection and placement of electrodes}

The selection and placement of the electrodes plays an important role for the results recorded. According to Ohm's law, the intensity of the current flowing through a conductor is proportional to the voltage at its ends and inversely proportional to the resistance of the circuit. Therefore, in order to create more favorable conditions for the transmission of the electric potential specific procedures are required.

Proper skin preparation is required for better signal transmission from the muscle to be detected.

The information obtained describes the time interval between nerve pulses, the firing rate, and the characteristics of muscle synchronization. The morphology of the shape of motor unit action gives information regarding the general condition of muscle fibres [48].

The placement of electrodes close to the muscle innervation gives lower intensity signals with delay time because the signal may regress [49]. In addition, placing the electrodes near the tendon is not recommended as mentioned. The recommended point is considered on the muscle mass (muscle belly) [49,50].

However, in bipolar measurements, the energy of the signal is generally underestimated when electrodes are placed on motor end-plates, or the muscle-tendon junctions.

\section{Conclusion}

The application of SEMG Biofeedback requires the patient to cooperate actively. Specifically, when the grade of muscle strength is 1 or 2 according to the MRC (Medical Research Council) Muscle Power Grading, then the patient can participate in a biofeedback programme. The proposed treatment time is between $10-70 \mathrm{~min}-$ utes. After the program session, muscle fatigue and inability to concentrate occur. The treatment time ranges from 14 to 131 days for at least once or twice per week. During the application of SEMG Biofeedback, some time is needed for adaptation ( 4 to 5 minutes) until the patient is able to realize a particular muscle twitch and repeat it $[51,52]$.

The areas related to the application of S.EMG BFB concern problem evaluation, pain, and muscle retraining. Specifically, Sella G., et al. 2003 reports that the use of SEMG Biofeedback is considered an important rehabilitation tool for patients with neuromuscular problems. With electromyography recording, the problem can be clearly defined locally, and the mechanism of the problem's pathophysiology can be understood. The definition of the problem and the precise description of the pathophysiological mechanism can lead to the selection of the appropriate therapeutic framework and programme. The SEMG BFB has two directions, diagnosis and differential diagnosis, treatment programme, assisting in the restoration of normal mobility and proprioception by re-recording and modifying existing pathological imprints [53].

Research by Pullman., et al. 2000 indicates that, although the surface electromyography is not a valid diagnostic tool because there are interfering factors (the tissues between the target muscle and the electrode, adjacent organs), it is considered a valid tool for assessing therapeutic interventional programmes. However, Geisser M., et al. 2005, in a meta-analysis research, claim that surface electromyography has the reliability required to compare functional or dysfunctional muscle contraction in case of low back pain [54].

In addition, SEMG Biofeedback yielded positive results in a research by Ladd HW., et al. 1981 on the evaluation of peripheral nerve reconstruction by measuring neuromuscular activity. Considering myoelectric signals is useful in indicating the occurrence of peripheral sprouting and axonal re-growth, of central reorganisation, as well as in indicating improved metabolic activity in a muscle. Myoelectric signals differentiated clearly between muscles where regeneration could be assumed to be in progress and muscles where it was not in progress. This differentiation could be made prior to the occurrence of any observable muscle contraction $[55,56]$.

SEMG biofeedback seems to be a useful tool for therapeutic approaches and assessment. Being aware of his or her slight muscular contractions, the patient mobilises himself or herself to make further contractions. The basic aims of SEMG biofeedback are to, increase maximum voluntary centrally mediated neuromuscular 
activity, dissociate desired and interfering neuromuscular activity and improve patient control over neuromuscular activity.

The use of SEMG has several distinct yet complementary aims according to the objectives of the research. Among these aims, three clinical reasons related to the use of SEMG have been identified: enhanced knowledge of the physiopathology of the disease, the diagnosis of the disease and the patient's evaluation and follow-up [51,56,57].

\section{Conflict of Interest}

There is no conflict of interest.

\section{Bibliography}

1. Galvani L. "Green RM, trans: Commentary on the Effect of electricity on muscular motion". Elizabeth Licht, Publisher (1953).

2. Criswell E. “Cram's introduction to surface electromyography second edition Jones and Bartlett Learning (2010).

3. Schwartz MF. "Andrasc Biofeedback: a practitioner's guide". New York: Guilford Press (2003).

4. Marinacci M Horande. "Electromyogram in Neuromuscular Re-education". Bulletin of the Los Angeles Neurological Society 25 (1960): 57-71.

5. Adams JA. "A closed-loop theory of motor learning". Journal of Motor Behavior 3.2 (1971): 111-149.

6. Chapman SL. "A review and clinical perspective on the use of EMG and thermal biofeedback for chronic headaches". Pain 27.1 (1986): 1-43.

7. Wolf SL and Binder-MacLeod SA. "Electromyographic biofeedback applications to the hemiplegic patient. Changes in lower extremity neuromuscular and functional status". Physical Therapy 63.9 (1983): 1404-1413.

8. Holtermann A., et al. "The use of EMG biofeedback for learning of selective activation of intra-muscular parts within the serratus anterior muscle A novel approach for rehabilitation of scapular muscle imbalance". Journal of Electromyography and Kinesiology 20.2 (2010): 359-365.

9. Cressman EK and Henriques DYP. "Sensory Recalibration of Hand Position Following Visuomotor Adaptation". Journal of Neurophysiology 102 (2009): 3505-3518.
10. Dunn TG., et al. "The learning process in biofeedback: Is it feed-forward or feedback?" Applied Psychophysiology and Biofeedback 11 (1986): 143-156.

11. La Croix JM. "The acquisition of autonomic control through biofeedback: The case against an afferent process and a two process alternative". Psychophysiology 18 (1981): 573-587.

12. O'Callagh ME and Couvadelli B. "Use of Self-Instructional Strategies with Three ameliorating Neurologically Impaired Adults Cognitive". Therapy and Research 22 (1998): 91-107.

13. James C Rosen., et al. "Cognitive behavior therapy for negative body image”. Behavior Therapy 20 (1989): 393-404.

14. De Craen AJ., et al. "Placebos and placebo effects in medicine: historical overview". Journal of the Royal Society of Medicine 92 (1999): 511-515.

15. Shapiro AK. "A historic and heuristic definition of the placebo". Psychiatry 964.27 (1964): 52-58.

16. Price Donald D., et al. "A Comprehensive Review of the Placebo Effect: Recent Advances and Current Thought". Annual Review of Psychology 59 (2008): 565-590.

17. Levine JD., et al. "The mechanism of placebo analgesia". Lancet 23 (1978): 654-657.

18. Benedetti F., et al. "Neurobiological Mechanisms of the Placebo Effect". The Journal of Neuroscience 25 (2005): 10390-10402.

19. Mayberg HS., et al. "The Functional Neuroanatomy of the Placebo Effect". The American Journal of Psychiatry 159 (2002): 728-737.

20. Bandura A. "Self-efficacy". In V. S. Ramachaudran (Edition.), Encyclopedia of human behavior New York: Academic Press. (Reprinted in H. Friedman [Edition.], Encyclopedia of mental health. San Diego: Academic Press 4 (1994): 71-81.

21. Haynes R B., et al. "Systematic review of randomised trials of interventions to assist patients to follow prescriptions for medications". Lancet 348 (1996): 383-386.

22. Greenfield S., et al. "Expanding patient involvement in care. Effects on patient outcomes". Annals of Internal Medicine 102 (1985): 520-528.

23. Alain Deccache. "Teaching, training or educating patients? Influence of contexts and models of education and care on practice in patient education Patient Education and". Counseling 26 (1995): 119-129. 
24. Rosenthal R and Jacobson L. "Pygmalion in the Classroom". New York: Holt Rinehart and Wilson (1968).

25. Enhancing Human Performance: Background Papers". Issues of Theory and Methodology (1988). Commission on Behavioral and Social Sciences and Education (CBASSE) Committee on Techniques for the Enhancement of Human Performance". National Research Council (1988).

26. Cronbach Lee Joseph and Snow Richard E. "Aptitudes and instructional methods: a handbook for research on interactions". Irvington Publisher Inc (1981).

27. Basmajian J. "Research foundation of EMG Biofeedback in Rehabilitation”. Biofeedback and Self-Regulation 13 (1988): 4.

28. Marinacci Horande M. "Electromyogram in Neuromuscular Re-education". Bulletin of the Los Angeles Neurological Society 25 (1960): 57-71.

29. Wolf LS. "Electromyographic Biofeedback Applications to Stroke Patients A Critical Review". Physical Therapy 63 (1983): 1448-1459.

30. Wolf IS and Binder-Macleod S. "Electromyographic Biofeedback Applications to the Hemiplegic Patient Changes in Upper Extremity Neuromuscular and Functional Status". Physical Therapy 63 (1983): 1393-1403.

31. Woolf CJ and Salter MW. "Neuronal Plasticity: Increasing the Gain in Pain”. Science 288 (2000): 1765-1769.

32. Elbert T., et al. "Increased Cortical representation of the fingers of the left hand in string players". Science 270 (1995): 305-307.

33. Pourrier Salima D., et al. "Three cases of referred sensation in traumatic nerve injury of the hand: implications for understanding central nervous system reorganization". Journal of Rehabilitation Medicine 42 (2010): 357-361.

34. Merzenich MM., et al. "Progression of change following median nerve section in the cortical representation of the hand in areas $3 \mathrm{~b}$ and 1 in adult owl and squirrel monkeys". Neuroscience 10 (1983): 639-665.

35. Merzenich MM and Jenkins WM. "Reorganization of cortical representations of the hand following alterations of skin inputs induced by nerve injury, skin island transfers, and experience". The Journal of Hand Therapy 6 (1993): 89-104.
36. Martinez M., et al. "Differential tactile and motor recovery and cortical map alteration after C4-C5 spinal hemisection". Experimental Neurology 221 (2010): 186-197.

37. Floyer-Lea A and Matthews PM. "Distinguishable Brain Activation Networks for Short- and Long-Term Motor Skill Learning". Journal of Neurophysiology 94 (2005): 512-518.

38. Su Jiang., et al. "Reorganization in motor cortex after brachial plexus avulsion injury and repair with the contralateral c7 root transfer in RATS". Microsurgery 30 (2010): 314-320.

39. Wall J T., et al. "Human brain plasticity: an emerging view of the multiple substrates and mechanisms that cause cortical changes and related sensory dysfunctions after injuries of sensory inputs from the body". Brain Research Reviews 39 (2002): 181-215.

40. Bayona NA., et al. "The role of task-specific training in rehabilitation therapies". Topics in Stroke Rehabilitation 12.3 (2005): 58-65.

41. Wolf S. "The First Basmajian Lecture: Reflections on John V. Basmajian: Anatomist, Electromyographer, Scientist". Journal of Electromyography and Kinesiology 7 (1997): 213-219.

42. De Luca C J Adam A., et al. "Decomposition of Surface EMG Signals". Journal of Neurophysiology 96 (2006): 1646-1657.

43. McGill K., et al. "EMGLAB: An interactive EMG decomposition program”. Journal of Neuroscience Methods 149 (2005): 121133.

44. DeLuca CJ and Merletti R. "Surface myoelectric signal crosstalk among muscles of the leg. Electroencephalogr". Clinical Neurophysiology 69 (1988): 568-575.

45. Farina D., et al. "Surface EMG Crosstalk valuated from Experimental Recordings and Simulated Signals Reflections on Crosstalk Interpretation, Quantification and Reduction Methods". Informatics Med 43 (2004): 30-35.

46. Solomonow M., et al. "Surface and wire EMG crosstalk in neighbouring muscles". Journal of Electromyography and Kinesiology 4 (1994): 131-142.

47. Kohei Watanabe a and Hiroshi Akima. "Cross-talk from adjacent muscle has a negligible effect on surface electromyographic activity of vastus intermedius muscle during isometric contraction". Journal of Electromyography and Kinesiology 19 (2009): 280-e289. 
48. CJ De Luca A Adam., et al. "Decomposition of Surface EMG Signals”. Journal of Neurophysiology 96 (2006): 1646-1657.

49. Kleine BU., et al. "Influence of motoneuron firing synchronization on SEMG characteristics in dependence of electrode position". Journal of Applied Physiology 91 (2001): 1588-1599.

50. Rainoldi A and Melchiorri G Caruso I. "A method for positioning electrodes during surface EMG recordings in lower limb muscles". Journal of Neuroscience Methods 134 (2004): 37-43.

51. Ladd HW., et al. "Evaluation and prediction of regenerative processes by quantitative analysis of the electromyographic response and biofeedback in peripheral nerve injuries". International Rehabilitation Medicine 3 (1981): 201-205.

52. Bilodeau M., et al. "EMG frequency content changes with increasing force and during fatigue in the quadriceps femoris muscle of men and women". Journal of Electromyography and Kinesiology 13 (2003): 83-92.

53. Sella GE. "Neuropathology Considerations: Clinical and SEMG/ Biofeedback Applications". Applied Psychophysiology and Biofeedback 28 (2003): 93-105.

54. SL Pullman., et al. "Clinical utility of surface EMG: Report of the Therapeutics and Technology Assessment Subcommittee of the American". Academy of Neurology 55 (2000): 171-177.

55. Geisser M., et al. "Meta- Analytic Review of Surface Electromyography Among Persons With Low Back Pain and Normal, Healthy Controls". The Journal of Pain 6 (2005): 711-726.

56. Ladd HW., et al. "Evaluation and prediction of regenerative processes by quantitative analysis of the electromyographic response and biofeedback in peripheral nerve injuries". International Rehabilitation Medicine 3 (1981): 201-205.

57. Woodford H and Price C. "EMG biofeedback for the recovery of motor function after stroke". Cochrane Database of Systematic Reviews 18 (2007): 2.

\section{Volume 4 Issue 9 September2021}

(C) All rights are reserved by Magdalini Stamou. 